\title{
¿SON LAS HABILIDADES MATEMÁTICAS UN ASUNTO DE GÉNERO? LOS ESTEREOTIPOS DE GÉNERO ACERCA DE LAS MATEMÁTICAS EN NIÑOS Y NIÑAS DE KÍNDER, SUS FAMILIAS Y EDUCADORAS ${ }^{1}$
}

\author{
María Francisca del Río ${ }^{2}$ \\ Katherine Strasser ${ }^{3}$ \\ María Inés Susperreguy ${ }^{4}$
}

\begin{abstract}
RESUMEN
En Chile se observa una importante brecha en el logro de matemática a favor de los hombres, tanto en pruebas nacionales como internacionales. Algunas teorías plantean que al menos una parte de estas diferencias podrían ser atribuibles a estereotipos de género relativos a las habilidades matemáticas. Este estudio explora los estereotipos de género, implícitos y explícitos, de estudiantes, madres, padres y educadoras en Santiago de Chile. Participaron 180 niños y niñas de medio-bajo y alto NSE (87 niñas) de kínder, sus padres y las 19 educadoras de sus salas, pertenecientes a colegios y escuelas del área urbana de Santiago, Chile. Se aplicaron medidas de estereotipos explícitos e implícitos a los niños, madres, padres y educadoras. Los resultados mostraron que, en promedio, tanto los adultos como los niños del estudio sostienen un estereotipo que asocia la matemática con el género masculino. En el caso de los niños, los resultados de medidas implícitas mostraron que todos los niños y las niñas de NSE medio-bajo asocian la matemática con lo masculino. Las niñas de NSE alto no asocian la matemática con ningún género. Estos hallazgos sugieren que los estereotipos de género y matemática podrían constituir un factor en las brechas de género en términos de rendimiento matemático observadas en nuestro país y abren posibilidades para el desarrollo de intervenciones y políticas públicas tendientes a ofrecer iguales oportunidades de aprendizaje a niños y niñas, específicamente en los grupos de niñas y madres de NSE medio-bajo, quienes presentan de manera más intensa el estereotipo.
\end{abstract}

Palabras clave: estereotipos, género, kínder, medidas implícitas, rendimiento matemático.

1 Este estudio se pudo realizar gracias al apoyo del Consejo Nacional de Educación (CNED) a través de su Convocatoria de apoyo a la investigación en educación y del Fondo Nacional de Ciencia y Tecnología (Fondecyt), a través del proyecto No 1150156.

2 Facultad de Educación, Universidad Diego Portales, Santiago, Chile. Contacto: francisca. delrio@mail.udp.cl

3 Escuela de Psicología, Facultad de Ciencias Sociales, Pontificia Universidad Católica de Chile, Santiago, Chile. Contacto: kstrasse@uc.cl

4 Facultad de Educación, Pontificia Universidad Católica de Chile. Contacto: misusper@uc.cl 


\title{
IS MATH ABILITY A GENDER ISSUE? GENDER STEREOTYPES ABOUT MATH IN KINDERGARTEN CHILDREN, THEIR FAMILIES AND TEACHERS
}

\begin{abstract}
National as well as international standardized assessments reveal that Chile has a large gender gap in math achievement that favors male students. Some theories propose that at least part of this gap can be explained by the effects of gender stereotypes that refer to abilities in math. In the present study, we examine the implicit and explicit gender stereotypes related to math of kindergarten children, their mothers, fathers and teachers in Santiago, Chile. The participants were 180 kindergarteners from mid-low and high SES (87 girls and 93 boys, from 19 classrooms), their parents and classroom teachers, from the urban area of Santiago, Chile. Participants were assessed on explicit and implicit gender stereotypes on math ability. Results show that, on average, both adults and children hold a stereotype that associates math to the male gender. In the case of children, the results of the implicit assessments reveal that all of the boys and mid-low-SES girls relate math to the male gender, while high-SES girls do not associate math to any gender. These findings suggest that math and gender stereotypes could help explain part of the math gender gap present in Chile, and thus suggest opportunities for early interventions and public policies that seek to offer children equal learning opportunities, particularly for girls and mothers from mid-low SES, who most intensely present this stereotype.
\end{abstract}

Keywords: gender, kindergarten, implicit measures, math achievement, stereotypes.

\section{Introducción}

En Chile se observa una importante brecha en el logro de matemática a favor de los hombres, la que también se expresa en las elecciones de una carrera profesional (Organisation for Economic Cooperation and Development, OECD, 2010; 2014). Los resultados de PISA 2009 y 2012 muestran que, mientras en otros países latinoamericanos se observan pequeñas diferencias a favor de los hombres en el test de matemática (por ejemplo Uruguay), en Chile los hombres obtienen un rendimiento significativamente mayor al de las mujeres (esta diferencia es solo menor a la observada en Colombia). Este patrón también se aprecia en las pruebas estandarizadas de matemática chilenas (Sistema Nacional de Evaluación de Resultados de Aprendizaje, Simce, 2012; 2013). Un fenómeno similar se evidencia en el test de selección universitaria (PSU), donde los hombres sistemáticamente logran mejores puntajes en matemática que las mujeres (Ministerio de Educación de Educación de Chile, Mineduc, 2011). 
Estas diferencias afectan la elección femenina de carreras universitarias ligadas con las áreas de ciencia/tecnología/matemática (STEM en inglés), y su acceso a trabajos mejor remunerados, los que no se distribuyen de manera equitativa entre los géneros (Consejo Nacional de Educación, CNED, 2005). En efecto, a lo largo de los últimos 25 años menos del $20 \%$ de los estudiantes chilenos que se inscribieron en carreras de corte tecnológico fueron mujeres (Blázquez, Álvarez, Bronfman y Espinosa, 2009).

En nuestro país también se presenta de manera repetida una importante brecha de nivel socioeconómico (NSE) en los resultados de las pruebas estandarizadas en todas la áreas evaluadas, incluyendo matemática (Simce, 2012; 2013). Los resultados oficiales de estas pruebas no llevan a cabo un análisis que muestre si es que la brecha de género en matemática se distribuye de manera distinta entre las niñas y niños de diferente NSE. Sin embargo, un análisis propio sobre la base de los resultados Simce de Matemática 2011 de octavo básico mostró que, si bien existe una diferencia significativa en este puntaje entre las niñas y niños de NSE tanto alto como bajo, la brecha de género es dos veces mayor en NSE bajo que en NSE alto.

Lo anterior se confirma en estudios internacionales que han mostrado que el NSE impacta diferenciadamente a hombres y mujeres respecto de su logro académico y elección posterior de área de estudios terciarios, siendo las mujeres más pobres quienes, en comparación con las de mayor NSE, muestran mayores problemas en matemática y eligen con menor probabilidad carreras en esa área (Ware \& Lee, 1986). Resultados similares obtuvo una investigación en nuestro país realizada en algunas comunas de la VII y VIII Región, donde los datos no solo mostraron que los resultados bajos y medio-bajos de matemática del Simce de cuarto básico se concentraban con mayor frecuencia entre las niñas de NSE bajo, sino que la brecha entre niños y niñas es mayor mientras el NSE es menor (Madero, 2011). El conjunto de estos datos muestra que serían las niñas de NSE bajo quienes tienen la mayor probabilidad de presentar problemas en matemática, más que los niños y más que las niñas de otros niveles socioeconómicos. Esto justifica poner un foco especial en este grupo. 
La diferencia en el desempeño matemático entre niños y niñas es un fenómeno que puede ser explicado desde diversas perspectivas (genética, acceso a oportunidades, estereotipos culturales, por nombrar algunas). En este estudio el problema se enfoca desde el punto de vista de los estereotipos y su efecto en el desempeño académico. Al respecto, la psicología social y las teorías acerca de la motivación sugieren que los estereotipos de género pueden influenciar significativamente los comportamientos, tanto de profesores, como de niñas y niños, afectando su desempeño y aprendizaje (Gunderson, Ramírez, Levine \& Beilock, 2011; Herbert \& Stipek, 2005; KurtzCostes, Rowley, Harris-Britt \& Woods, 2008; Mizala, Martínez \& Martínez, 2015). En este caso, el estereotipo "las niñas no son buenas para la matemática" es uno que tiene alta aceptación en el mundo (Nosek, Smyth, Sriram \& Lindner, 2009).

En contraste con gran parte de la investigación en esta área -que ha indagado en niños escolares y universitarios y sus profesores-, el presente estudio se focaliza en los niños y niñas de educación parvularia, sus educadoras y familias, un grupo que ha sido raramente estudiado respecto de estas creencias (Cvencek, Meltzoff \& Greenwald, 2011; del Río \& Strasser, 2013; Gunderson et al, 2011). El supuesto tras el foco en niños de esta edad es que las creencias y atribuciones que pueden contribuir a un logro diferenciado en matemática de niños y niñas comienzan a formarse temprano en el desarrollo, a partir de mensajes de los medios de comunicación y las conductas de cercanos (Coyne, Linder, Rasmussen, Nelson \& Collier, 2014; Tiedemann, 2002). Además, es en esta etapa cuando estas creencias podrían ser más fácilmente modificables (Cvencek, Kapur \& Meltzoff, 2015; Cvencek, Meltzoff \& Greenwald, 2011; Gunderson et al., 2011).

Para entender el origen de los estereotipos de género y matemática, los estudios del área han tenido como foco los padres y profesores, quienes se considera representan las mayores influencias ambientales en el desarrollo de los niños, por lo que resultan centrales en la formación de actitudes dirigidas a lo académico (Gunderson et al., 2011; Tiedemann, 2000b). Por esto, si se busca entender los estereotipos de los niños es central conocer también los de los adultos más relevantes en su vida, como son los padres y profesores. 
En suma, lo anterior conduce a la necesidad de conocer si los niños chilenos de kínder sostienen estereotipos relativos a habilidades matemáticas y el género y, cuál es la relación que estos estereotipos tienen con los que sostienen sus educadoras, madres y padres.

\section{Cognición social y estereotipos}

Un estereotipo es la generalización de características idénticas para un grupo de personas, sin importar la diferencia que pudiera existir entre los miembros de ese grupo (Aronson, Wilson \& Akert, 2010). Una vez que se forman, los estereotipos son resistentes al cambio, aunque las personas se enfrenten a nueva información que pueda ser contradictoria con estos. Adicionalmente, estereotipar es un proceso principalmente cognitivo, por lo que se considera que forma parte de la cognición social que las personas desarrollan a lo largo de sus vidas. La cognición social se refiere a cómo las personas se ven a sí mismas, a otras personas y a los grupos sociales a los que pertenecen. Los estereotipos son un aspecto de la cognición social que es utilizado, entre otras cosas, para ordenar el mundo y tomar decisiones acerca de situaciones sociales (Baron, Schmader, Cvencek \& Meltzoff, 2014).

Los estereotipos pueden operar a un nivel consciente (conocimiento explícito) y también a un nivel no consciente (conocimiento implícito). Esto supone que la información que está disponible de manera consciente es solo una pequeña porción de lo que ocurre en el cerebro de las personas y puede ser irrelevante para entender sus acciones o decisiones (Nosek et al., 2011). Greenwald y Banaji (1995) plantearon el término "cognición social implícita" para referirse a los procesos cognitivos que ocurren fuera de la capacidad o control consciente.

Para todas las culturas, el desarrollo de la concepción de género entre los niños ocurre tempranamente y suele ser más sobresaliente que las categorías de raza, de etnia, de religión o de nacionalidad (Bigler \& Liben, 2007; Levy \& Killen, 2008; Martin \& Ruble, 2004; Martin, Ruble \& Szkrybalo, 2002). Tanto es así que muy rápidamente, una vez que aprenden a categorizar a otros sobre la base del género, los niños y niñas comienzan a asociar diferentes rasgos y actitudes con 
hombres y mujeres. Estas asociaciones se vuelven implícitas temprano en el desarrollo y se relacionan con una selección de conductas que llevan a hombres y mujeres a diferentes roles y actividades (Davies, Spencer \& Steele, 2005; Steele, Spencer \& Aronson, 2002).

\section{Estereotipos infantiles acerca del género y el desempeño académico}

Diversas investigaciones dan cuenta de que los niños sostienen desde temprano estereotipos referidos al desempeño académico de niños y niñas. Meece, Glienke y Burg (2006) mostraron que los niños de los EE.UU. ya tienen en los primeros años de la educación básica diferentes creencias acerca de sus habilidades. Estos comenzarían la educación formal con una alta percepción respecto de sus habilidades matemáticas, mientras que las niñas con una alta percepción de sus habilidades lingüísticas y artísticas. Otro ejemplo de esto son los resultados de Galdi, Cadinu y Tomasetto (2014) con niños italianos, que mostraron que ya a la edad de seis años las niñas sostenían estereotipos que asociaban la matemática a los hombres lo que, a su vez, se correlacionaba con un menor rendimiento en esta disciplina. Otras investigaciones sugieren que estas creencias tempranas no se explican por el desempeño escolar real. Herbert y Stipek (2005) entrevistaron aproximadamente a 300 niños entre $1^{\circ}$ y $5^{\circ}$ año de educación básica y encontraron que, a pesar de que las niñas puntuaban más bajo sus propias capacidades matemáticas en relación con los niños, el desempeño matemático no difería estadísticamente entre ambos grupos. Estos estereotipos no solo se mantienen, sino que se profundizan a medida en que se avanza en la educación formal. Estudios realizados entre preadolescentes y adolescentes muestran que el autoconcepto, las expectativas de logro y la motivación en matemática de los niños son significativamente mayores que los de las niñas (Berg \& Klinger, 2009; Skaalvik \& Skaalvik, 2004).

En el país existe poca investigación en esta área. Una excepción es un estudio del año 2014, que se preguntó si, dada la brecha de género en rendimiento matemático en la adolescencia, las habilidades matemáticas eran ya distintas para niños y niñas en el primer ciclo de educación básica. Luego de aplicar pruebas que medían competencias 
numéricas, los resultados no mostraron diferencias entre niños y niñas de seis y siete años de edad (Cerda, Pérez \& Ortega-Ruiz, 2014). Otro estudio nacional relevante es uno comisionado en 2008 por el Servicio Nacional de la Mujer (Sernam), dirigido a examinar las interacciones, desde una perspectiva de género, entre los profesores y estudiantes en escuelas públicas. Los investigadores analizaron videos de clases que incluían de manera proporcional profesores hombres y mujeres, y clases de $5^{\circ}$ y $8^{\circ}$ básico. Entre otras cosas, el estudio mostró que los profesores (de ambos géneros) interactuaban con más frecuencia con los niños que con las niñas, y que esta diferencia no se explicaba por una mayor frecuencia de preguntas y comentarios espontáneos de los niños. También se observó la tendencia de los profesores a ser más paternalistas y a ofrecer más ayuda a las niñas en áreas como la matemática; y la tendencia a utilizar ejemplos con estereotipos de género para ilustrar conceptos (por ejemplo, "¿qué es lo que una mamá necesita hacer al escribir la lista de supermercado?; o "un chofer de camiones maneja $50 \mathrm{~km}$..."). Los padres chilenos también expresan actitudes asociadas con estos estereotipos. En la última evaluación PISA en Chile el 50\% de los padres de niños de 15 años reportaron que esperaban que sus hijos trabajaran en el área de ciencia/tecnología/ matemática, lo que contrasta con solo un $16 \%$ de esa preferencia para las niñas de 15 años (OECD, 2015).

Otro estudio en Chile se abocó a conocer las representaciones que los propios estudiantes tienen de sus habilidades académicas. Blázquez, Álvarez, Bronfman y Espinosa (2009) midieron el autoconcepto matemático de 1.697 estudiantes de secundaria, encontrando que las niñas tenían un autoconcepto marcadamente menor que los niños. Asimismo, observaron que el autoconcepto matemático fue más importante al determinar la carrera a estudiar, que el apoyo académico de padres y profesores.

El único estudio chileno publicado acerca de las creencias diferenciadas por género respecto de las habilidades matemáticas en niños de edad preescolar mostró que ya a los cinco años de edad los niños entrevistados, sin diferencia de género, sostienen la creencia de que las niñas tienen una mayor probabilidad de no gustarles las matemática, de encontrarlas difíciles y de obtener 
un menor rendimiento en esta área del conocimiento (del Río \& Strasser, 2013).

\section{Efectos de las creencias y estereotipos}

La psicología social y las teorías en torno a la motivación sugieren que los estereotipos de género pueden influenciar significativamente los comportamientos académicos de las niñas y mujeres. La teoría de la amenaza del estereotipo de Steele y Aronson (1995) justamente plantea que en situaciones que activan los contenidos negativos de los estereotipos, los grupos afectados actúan de acuerdo con lo que se espera de ellos. Así las niñas, frente a un test de matemática, rendirán menos que los niños, pues se ajustarían a lo que el estereotipo espera de ellas.

Lamentablemente, las creencias estereotipadas acerca del género se pueden encontrar temprano en el desarrollo (Alexander, Wilcox \& Woods, 2009; Ambady, Shih, Kim \& Pittinsky, 2001; Cherney \& London, 2006; Maccoby, 2002), y la evidencia muestra que activar estas creencias puede afectar el desempeño académico (Ambady et al., 2001; Nosek et al., 2009). En efecto, un metaanálisis que combinó los datos de 18.876 estudiantes de cinco países mostró que la sola condición de las pruebas estandarizadas de matemática funciona como una amenaza activando los estereotipos de género en las niñas, lo que a su vez determina que este tipo de evaluaciones subestime su habilidad matemática real (Walton \& Spencer, 2009).

En consonancia, numerosos estudios han mostrado que la percepción subjetiva de las propias habilidades (autoconcepto académico) influenciada por los estereotipos de género, juega un rol importante en el logro académico (por ejemplo, Fredricks \& Eccles, 2002; Leahey \& Guo, 2001; Marsh, Trautwein, Lüdtke, Köller \& Baumert, 2005), y que este efecto explica en parte la brecha de género en matemática y ciencia durante la educación básica (Herbert \& Stipek, 2005; Kurtz-Costes et al., 2008), educación media y universitaria (Kiefer \& Sekaquaptewa, 2007). Pero la investigación en el área de las expectativas de desempeño académico diferenciadas por género se ha limitado típicamente a muestras de niños y niñas en 
edad escolar y adultos, y mayormente a poblaciones norteamericanas y europeas (Cvencek, Meltzoff, \& Greenwald, 2011; Gunderson et al., 2011).

\section{Origen de los estereotipos de género en matemática}

Es sabido que los estereotipos de género pueden ser transmitidos a través de los mensajes de los medios de comunicación, de los programas de televisión (Coyne et al., 2014), o a través de los mensajes verbales y no verbales de las personas con las que los niños interactúan (Nosek et al., 2009). Específicamente respecto de los estereotipos de género y matemática, a fin de entender su origen, los estudios han tenido como foco a los padres y profesores, quienes se considera que representan las mayores influencias ambientales en el desarrollo de los niños y, por ende, resultan centrales en la formación de actitudes dirigidas a lo académico (Gunderson et al., 2011; Tiedemann, 2000b).

En primer lugar, este cuerpo de estudios (todos realizados en naciones del hemisferio norte) muestra que las expectativas de padres (madres y padres) y profesores acerca de la competencia matemática de los niños son generalmente sesgadas y hacen una diferencia entre niños y niñas (entre otros, Jacobs, 1991; Schwartz \& Sinicrope, 2013; Tiedemann, 2000b). Por ejemplo, un estudio alemán con niños de $3^{\circ}$ y $4^{\circ}$ grado mostró que los padres de varones creían que sus niños eran más competentes en matemática, que los padres de niñas (Tiedemann, 2000b). Respecto de los profesores, estudios con docentes de primer grado (en EE.UU.) mostraron que estos perciben que sus mejores estudiantes varones eran más lógicos, más competitivos, más independientes en matemática y gustaban más de este ramo, que sus mejores estudiantes mujeres (Fennema, Peterson, Carpenter \& Lubinski, 1990). De manera similar, profesores de $3^{\circ}$ a $5^{\circ}$ grado en Alemania sostienen que los niños varones tienen una mayor habilidad matemática que las niñas, que los niños son más capaces en el pensamiento lógico que estas y que el ramo de matemática presenta un mayor desafío para las niñas que para los niños (Tiedemann, 2000a; 2000b; 2002). En nuestro país, un estudio realizado con profesores en formación mostró que la ansiedad matemática que estos futuros docentes tenía sesgaba sus expectativas 
respecto de los estudiantes y que estas expectativas eran menores si sus estudiantes era niñas (Mizala et al., 2015).

En segundo lugar, los estudios también muestran que las creencias de los padres y profesores respecto de las habilidades matemáticas de sus hijos/estudiantes predicen cómo los mismos niños evalúan sus propias capacidades, aún con mayor fuerza que su propio desempeño académico. Esto, subsecuentemente, afecta sus conductas y desempeño en matemática (Jacobs, 1991). Así, un estudio intercultural de padres e hijos en Taiwán, Japón y EE.UU. encontró que las madres de niños de kínder de estos tres países creían que los niños eran mejores en matemática que las niñas (Lummis $\&$ Stevenson, 1990). En la misma muestra, consecuentemente, los niños mostraron expectativas más altas que las niñas respecto de su éxito en matemática en $5^{\circ}$ grado. Este mismo fenómeno fue hallado en el marco de la relación entre los profesores y sus estudiantes. De este modo, un estudio realizado en Suiza sugirió que las expectativas de los profesores tuvieron un efecto $(p<, 001)$ en las actitudes en matemática diferenciadas por género (Keller, 2001). En otro tipo de acercamiento a este fenómeno, un estudio realizado en los EE.UU. con padres de niños de kínder a $2^{\circ}$ grado documentó una relación entre las creencias de los padres y la manera en que estos promovían actividades matemáticas en el hogar (Sonnenschein et al., 2012). Estos datos resultan especialmente relevantes, pues se ha demostrado una relación directa entre la estimulación matemática en el hogar y el logro académico posterior (LeFevre et al., 2009; Tudge \& Doucet, 2004). Así, estudios como el de Sonneschein y colaboradores (2012) sugieren un posible mecanismo por el cual las creencias de los padres podrían moldear las habilidades y rendimiento matemático de sus hijos.

La suma de estas investigaciones sugiere un modelo donde los estereotipos de padres y profesores sesgan su visión acerca de las habilidades matemáticas de sus propios hijos o estudiantes, lo que a su vez tiene un impacto significativo en el autoconcepto de los niños y niñas, y por consiguiente, en sus conductas y desempeño en matemática. 


\section{Medición de estereotipos de género en matemática}

Los estereotipos pueden ser medidos de manera explícita o implícita. La aplicación de pruebas explícitas supone que quien las responde está al tanto de qué es lo que se está midiendo, al solicitársele respuestas mediante autorreporte. En contraste, las medidas implícitas no consideran la forma de un autorreporte y no se requiere que el sujeto evaluado sea consciente de lo que se mide (Cvencek, Greenwald \& Meltzoff, 2011; Cvencek et al., 2015). Numerosas investigaciones utilizan ambas pues aportan distintos tipos de información. Las medidas explícitas e implícitas suelen correlacionarse, aunque de manera débil (por ejemplo, Cvencek, Meltzoff \& Greenwald, 2011; Fernández, Quiroga, Escorial \& Privado, 2014; Hofmann, Gawronski, Gschwender, Le \& Schmitt, 2005).

\section{a. Medidas explícitas}

Cuando se pregunta de manera directa, generalmente las personas niegan sostener estereotipos de género (Cvencek, Meltzoff \& Kapur, 2014), a pesar de que sea probable que los tengan. La negación ocurriría pues el entrevistado evalúa que estos estereotipos serían inapropiados socialmente (Herbert \& Stipek, 2005), o porque simplemente no sabe que albergan esos estereotipos. Por su parte, en niños pequeños existe adicionalmente el problema de que es posible que, por sus destrezas lingüísticas limitadas, no interpreten correctamente lo que se les pregunta o no sean capaces de entregar una respuesta adecuada. Este tipo de estudios son escasos con muestras de niños preescolares (Gunderson et al., 2011; Lindberg, Linkersdörfer, Ehm, Hasselhorn \& Lonnemann, 2013).

\section{b. Medidas implícitas}

Las medidas implícitas son menos susceptibles a la deseabilidad social que puede distorsionar las respuestas a los test de estereotipos (Greenwald et al., 2002). Para medir las representaciones implícitas, los test solicitan respuestas rápidas que reflejen procesos más automáticos e implícitos. Estas pruebas se basan en el supuesto de que los estereotipos pueden ser activados sin la atención o control del sujeto evaluado. 
Las pruebas implícitas se originaron dentro de la psicología social, pero en años recientes se han aplicado en estudios de las áreas de psicología cognitiva (Fazio \& Olson, 2003), psicología clínica (Teachman, Gregg \& Woody, 2001) y psicología del desarrollo (Cvencek et al., 2014; Dunham, Baron \& Banaji, 2006; Rutland, Cameron, Milne \& McGeorge, 2005). Estas pruebas son ejercicios computarizados de categorización, dirigidos a medir la fuerza de las asociaciones entre conceptos. Este formato permite estimar la preferencia de un concepto (por ejemplo, matemática) por sobre otro (por ejemplo, lectura), al asociarlo a un género (niño o niña).

La literatura ha mostrado que tanto en adultos (Greenwald et al., 2002; Herbert \& Stipek, 2005), como en niños (Cvencek et al., 2011; Cvencek, Meltzoff et al., 2011) estas pruebas implícitas han relevado con mayor sensibilidad que las medidas explícitas, datos acerca de estereotipos de género en general y de estereotipos de género y matemática, en particular. Por ejemplo, Cvencek, Meltzoff y Greenwald (2011) aplicaron pruebas implícitas -en este caso pruebas llamadas IAT (Implicit Association Test) - a niños y niñas estadounidenses entre 7 y 10 años de edad, para conocer si sostenían estereotipos que asociaran las habilidades matemáticas a un género particular. Sus hallazgos muestran que la idea de que "las matemáticas son para los niños", se encuentra ya presente en los niños y niñas desde segundo grado.

En Chile no existen experiencias de aplicación de medidas de IAT para medir estereotipos en niños preescolares, pero sí estudios en estudiantes mayores respecto de actitudes implícitas acerca de grupos minoritarios (Siebler et al., 2010), hacia la discapacidad cognitiva (Sirlopú et al., 2012), o hacia los homosexuales (Cárdenas y Barrientos, 2008). En consecuencia, en el presente estudio se propone aplicar medidas de estereotipos de corte explícito e implícito, con el fin de lograr identificar de mejor manera el fenómeno, focalizándose en una población preescolar, donde no se ha indagado masivamente acerca del mismo.

El objetivo del estudio es conocer los estereotipos de género relativos a habilidades matemáticas en niños y niñas de kínder de 
diferentes NSE, sus educadoras, madres y padres, utilizando medidas explícitas e implícitas. Adicionalmente se buscó establecer el nivel de relación entre las creencias explícitas e implícitas de los niños, niñas y las de los padres y educadoras. Como hipótesis se esperaba que todos estos actores asociaran matemática con mayor fuerza al género masculino, tanto en los test explícitos como en los implícitos y, que esta asociación fuera más fuerte en el NSE más bajo. Además se esperaba que ambos tipos de mediciones (explícitas e implícitas) correlacionaran de manera positiva. Finalmente, se esperó que los datos de los niños y niñas se asociaran significativamente con los de sus padres y educadoras.

\section{Metodología}

\section{Participantes}

Participaron 19 aulas de kínder, donde en cada una se evaluó a la educadora a cargo del curso y entre 8-10 niños, niñas y sus padres. De este modo, en total participaron 180 niños y 87 niñas de kínder, sus padres (180 parejas de padres) y 19 educadoras. Los niños fueron reclutados a través de sus escuelas y colegios. Todas las escuelas se ubicaron en el área urbana de Santiago de Chile y tenían financiamiento municipal o privado y con una orientación laica y mixta, de modo de evitar variables que interfirieran con el objeto de estudio. Estos 99 niños (provenientes de 10 salas de kínder) pertenecían a NSE bajo o medio-bajo, de acuerdo con la clasificación del Mineduc de las escuelas. En la Tabla 1 se puede apreciar la información resumen acerca de la muestra. El grupo de padres incluyó principalmente padres biológicos de los niños, pero también otras figuras parentales que vivían en el hogar con los niños, como abuelos, parejas de las madres y hermanos mayores de edad ( $n=19$ casos).

Tabla 1. Características de la muestra

\begin{tabular}{l|c|c|c}
\hline & $N$ & Media Edad (años) & D.E. \\
\hline Niñas & 87 & 5,58 &, 41 \\
\hline Niños & 93 & 5,55 &, 35 \\
\hline Madres & 180 & 36,3 & 6,8 \\
\hline Padres & 180 & 38,9 & 8,5 \\
\hline
\end{tabular}




\section{Procedimientos}

Para acceder a la muestra, en primer lugar se comprometió la participación de las escuelas y colegios a través de los directores de los establecimientos. Luego se invitó a los padres y madres. Solo se contactaron las parejas de padres donde ambos estaban dispuestos a participar, dado que era condición del estudio contar con datos del padre y la madre de cada niño y niña. En el caso que no se contara con un padre biológico, se invitó a una figura parental relevante para que participara. Esta figura fue típicamente otro adulto masculino que vivía con el niño/a, como abuelo, nueva pareja de la madre, hermano mayor de edad, etc.

Una vez que los padres aceptaban, eran evaluados (típicamente en su domicilio) y firmaban la autorización para que sus hijos participaran. Ya autorizados los niños, sus evaluaciones se llevaron a cabo de manera individual en un lugar tranquilo de su escuela. Las educadoras también fueron evaluadas en las escuelas. Todas las evaluaciones se aplicaron utilizando tabletas electrónicas.

\section{Instrumentos}

Se utilizaron los instrumentos desarrollados en el instituto ILABS (Insitute for Learning \& Brain Sciences) de la Universidad de Washington (UW) por Cvencek, Meltzoff y Greenwald (2011), por los que se pagó una licencia de uso. Estos consistieron en dos tests, uno de estereotipos que asociaron género y habilidades académicas de manera explícita y otro de corte implícito (IAT). Para el funcionamiento correcto de los tests, también fue necesario comprar las licencias de uso del programa computacional Inquisit by Millisecond, que recopila los datos de respuesta.

Los test tenían un formato distinto para niños y adultos (padres y educadoras). Los primeros contenían imágenes (Figura 1) y los segundos, conceptos (Figura 2). Ambos se aplicaron de manera computarizada a través de tabletas. Se utilizaron cinco tabletas, todas iguales, con un sistema operativo de Windows 8 y un tamaño de pantalla de 8". Se eligió este tamaño pues resultaba amigable para el manejo de los niños y niñas. 
En los test explícitos (TEE) quien contesta debe marcar su nivel de acuerdo con una afirmación. La respuesta la entregaban presionando una zona de la pantalla de la tableta. En el caso de los tests de adultos, las afirmaciones eran del tipo "la matemática es femenina" o "el lenguaje es femenino". En este caso, al medir ambos ítems cosas diferentes, la confiabilidad de la escala como en todo es baja (Alfa de Cronbach = ,57). Para el caso de los niños, ellos debían decidir a quién le gusta más la matemática o leer: a Pedro o a Sonia. La confiabilidad de la escala reflejó también los efectos de preguntar por dos áreas distintas (Alfa de Cronbach = -,43). Para ambos casos, las respuestas se entregaban a través de Escalas de Likert (de siete puntos en el caso de los adultos y de cuatro en el caso de los niños).

En los IAT la persona que responde debe categorizar estímulos asociándolos por pares. De la velocidad de respuesta al parear estímulos se derivan las creencias implícitas que los sujetos sostienen (Greenwald, McGhee \& Schwatz, 1998). A continuación, en la Figuras 1 y 2 se puede apreciar un ejemplo de las pruebas implícitas para niños y adultos. En la Figura 1 el ejercicio consiste en parear la imagen central con los estímulos asociados a fotos de niños o niñas. En este caso particular, la respuesta correcta es parear la imagen de matemática a los estímulos de la derecha de la pantalla. Si la imagen central fuera una representativa de lenguaje, la respuesta correcta sería la opuesta. Si existiera un estereotipo que asociara matemática a lo masculino, se esperaría una respuesta de asociación más lenta cuando, como en el caso de la Figura 1, la imagen de matemática está asociada con las niñas, que cuando estuviera asociada con los niños. 
Figura 1. Imagen de Test de Estereotipos Implícitos de Niños/as (IAT)
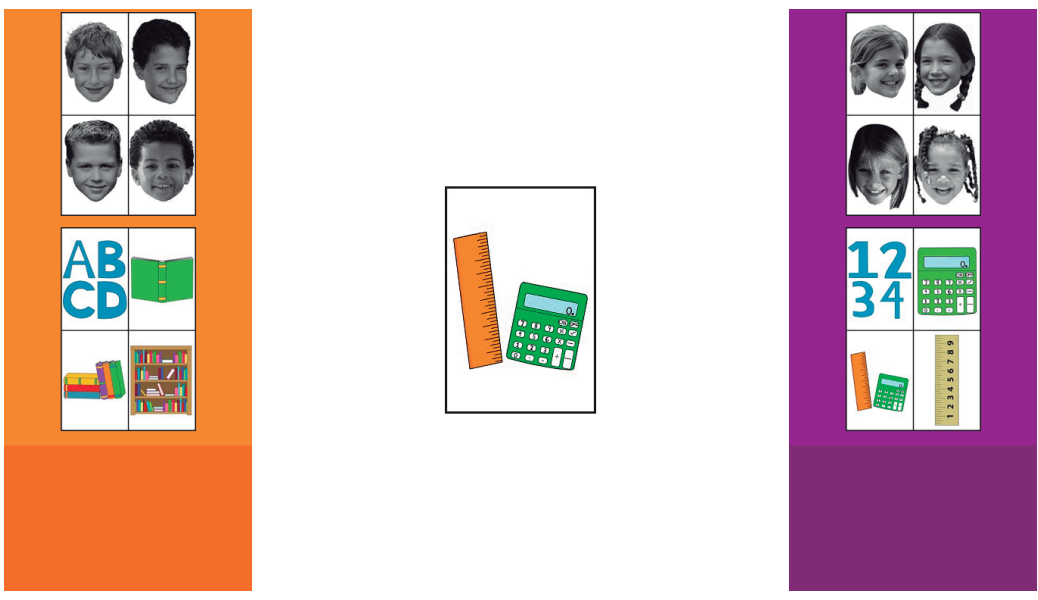

Fuente: University of Washington.

Los instrumentos fueron piloteados en Chile y perfeccionados antes del levantamiento de datos. Como resultado de este proceso se modificaron los tamaños de algunas figuras para hacerlas más visibles, y también algunos conceptos de los IAT de adultos que resultaban confusos. Estos últimos fueron cambiados por otros de uso más común.

En el caso de la Figura 2, el ejercicio es el mismo, pero para adultos. En este caso particular el ejemplo muestra en el centro un concepto asociado a lenguaje. El test implícito de estereotipos (IAT) siguió el típico esquema de estos test que contiene siete bloques. Del total de bloques, cinco corresponden a entrenamiento (1, 2, 3, 5 y 6) y, dos a los tests propiamente tales ( 4 y 7 ).

En el bloque 1, la tarea es discriminar entre imágenes (conceptos) representativos de matemática o lenguaje. En el bloque 2 se discriminan fotos (o conceptos) asociadas con lo femenino o masculino. En el bloque 3 se combinan los estímulos precedentes a modo de ejercicio y los mismos estímulos aparecen en el bloque 4 , pero estas respuestas son el primer test. En el bloque 5 se altera el orden de presentación de los estímulos del bloque 1, y el bloque 6 es una nueva práctica. Finalmente, el bloque 7 presenta nuevamente 
combinaciones de categorías, pero en orden invertido y considera el segundo test. La aplicación de los test y de los ítems que formaban parte de ambos tipos de test fueron contrabalanceados, como también el orden que aplicaron los test explícitos e implícitos.

Figura 2. Imagen de Test de Estereotipos Implícitos de Adultos (IAT)

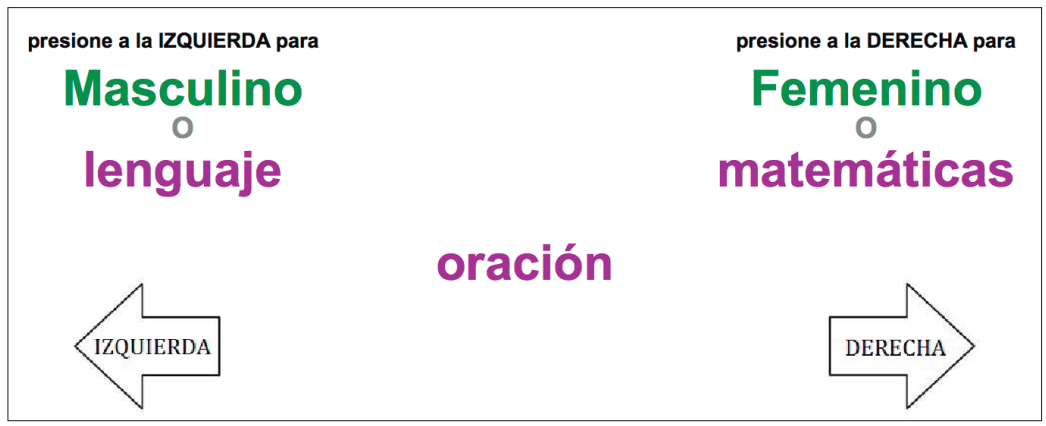

Fuente: University of Washington.

\section{Resultados}

Los datos fueron procesados por programas de análisis estadístico (SPSS 20.0). Se llevaron a cabo análisis descriptivos de los datos, inferenciales de comparación de grupos y de correlación. Para ambos tests y, tanto para el caso de adultos como el de los niños, los resultados se calcularon de forma que los puntajes positivos indicaran una asociación más fuerte entre matemática y el género masculino, mientras que los puntajes negativos indicaran una asociación entre matemática y el género femenino (Algoritmo D, Greenwald et al., 2003). De esta forma, una comparación estadística de los promedios alcanzados con el valor 0 (cero) podría dar cuenta de la dirección de la respuesta.

Antes de realizar los análisis, se descartaron los datos IAT que cumplieran con los siguientes criterios de exclusión: a) que tuvieran $10 \%$ o más de las respuestas de una velocidad de 300 ms o mayor; b) un ratio de error de respuesta de $35 \%$ o superior; o c) una latencia promedio de respuesta de 3 D.E. sobre la latencia promedio de respuesta de la muestra completa. Estos criterios excluyeron el total de 14 casos en el caso de los datos de los niños, 10 casos entre las 
madres y 8 casos entre los padres. Este procedimiento, consistente con los procedimientos de los IAT (Greenwald, Nosek \& Banaji, 2003), se realizó para reducir los datos erróneos y excluir las respuestas que se pudieran considerar como datos de valor atípico y que pudieran sesgar los resultados. De esta forma, finalmente los análisis IAT se realizaron con datos de 166 niños, 170 madres y 172 padres.

\section{Estereotipos de género en los niños y niñas}

Los resultados de los niños y niñas en el test de estereotipos explícitos (TEE) alcanzaron un promedio de $M=-, 04(D E=1,22)$. En el caso del test de estereotipos implícitos (IAT), el promedio fue de $M=, 02(D E=, 33)$. Al comparar ambos promedios con el valor 0 , no se observan diferencias significativas (TEE: $t(179)=-, 48, p=.626$; IAT: $t(166)=, 81, p=414)$. Esto refleja que los niños y niñas en general no muestran estereotipos que asocien la matemática con un género en particular, ni en el caso de las medidas explícitas, ni implícitas. En la Tabla 2 se pueden apreciar las medias en los test explícitos e implícitos diferenciadas por género y por NSE.

Tabla 2. Medias de niños y subgrupos de NSE en medidas explícitas e implícitas

\begin{tabular}{c|c|c|c|c|c|c|c|c}
\hline \multicolumn{1}{c|}{ Test } & \multicolumn{2}{|c|}{ Niño } & \multicolumn{2}{c|}{ Niña } & \multicolumn{2}{c|}{ NSE alto } & \multicolumn{2}{c}{ NSE bajo-m/bajo } \\
\hline & $M$ & $D E$ & $M$ & $D E$ & $M$ & $D E$ & $M$ & DE \\
\hline TEE &,$- 08 *$ & 1,24 &, 00 & 1,20 &, 08 & 1,14 &,- 14 & 1,27 \\
\hline IAT &, 07 &, 31 &,- 03 &, 34 &,- 02 &, 33 &, $05 *$ &, 32 \\
\hline
\end{tabular}

* Media estadísticamente distinta del valor $0 . p<, 05$.

Al observar los datos de niños y niñas por separado, se puede apreciar que no existen diferencias estadísticas en las respuestas del TEE, o sea niños y niñas contestan de igual forma en la medida explícita de estereotipos $(t(178)=, 47, p=, 638)$. Pero sí se presentan en el IAT $(t(165)=-1,9, p<, 05)$, donde los niños tienen como promedio una respuesta positiva (distinta estadísticamente del valor cero, $t(84)=2,06, p<, 05)$ que muestra una tendencia a asociar la matemática al propio género. Por su parte el promedio de las niñas en el IAT no es distinto del valor cero $(t(81)=-, 79, p=, 638)$. Esto se puede observar con mayor claridad en el Gráfico 1, donde se 
aprecia que las cajas correspondientes a las respuestas de los niños se ubican, en su mayoría, por sobre el valor 0; mientras las niñas, en su mayoría, cercanas a ese valor.

Gráfico 1. Resultado de medidas implícitas de estereotipos en niñas y niños

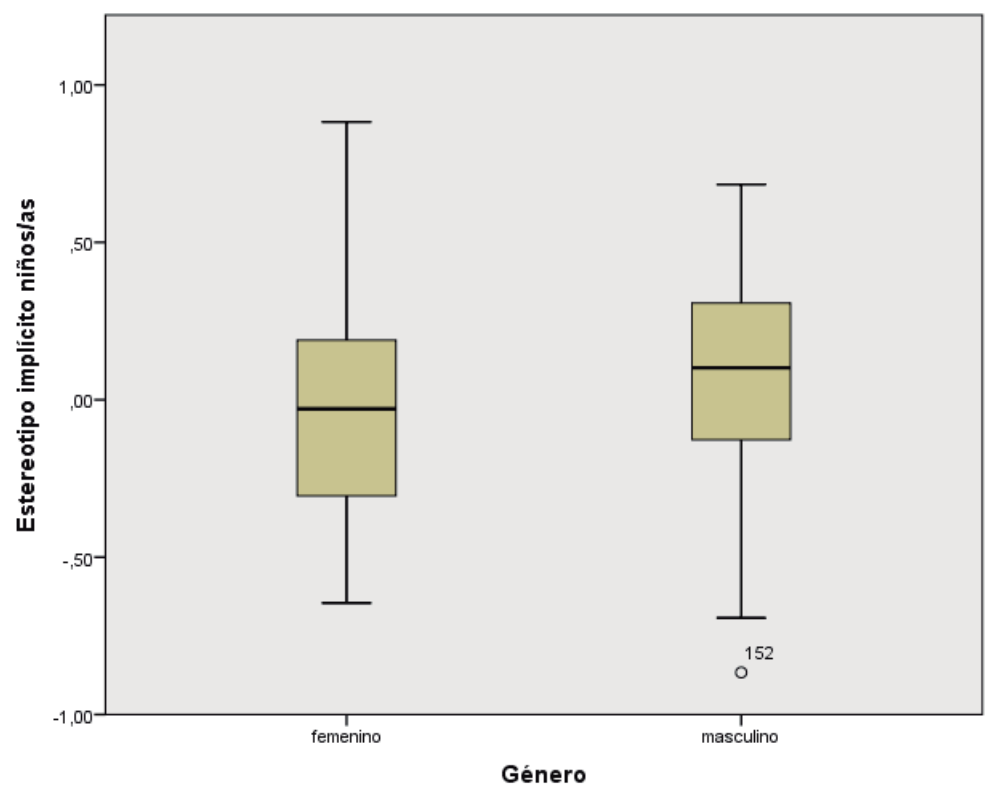

Por otra parte, si se analizan los resultados por grupo de NSE se obtiene que no existen diferencias estadísticas al compararlos en los resultados del TEE $(t(178)=-1,26, p=, 209)$, pero sí en los del IAT $(t(165)=1,58, p<, 05$, unilateral), donde se puede apreciar que los niños y niñas de NSE alto tienen una tendencia de respuesta que no es diferente estadísticamente del valor 0 (o sea, que no asocian matemática a un género específico), mientras que los niños y niñas de NSE bajo asocian la matemática a lo masculino $(t(88)=1,71$, $p<, 05$, unilateral). 
Gráfico 2. Resultado de medidas implícitas de estereotipos de niños/as de acuerdo con NSE

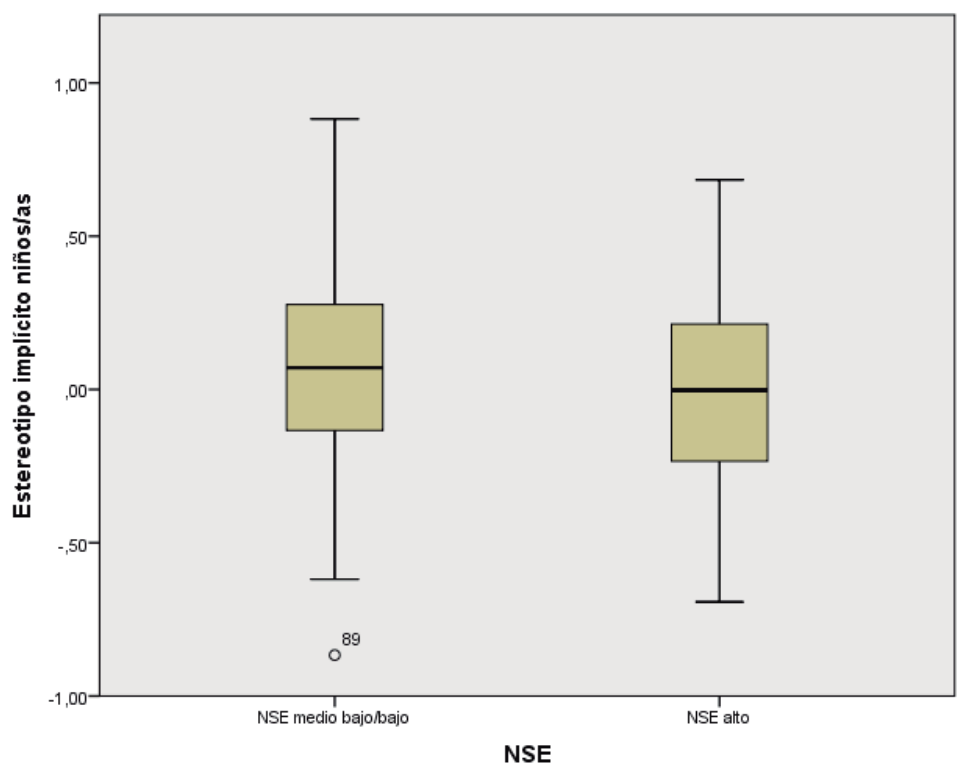

Los resultados anteriores se confirman al realizar un análisis de varianza factorial (género*NSE), donde la interacción de ambas variables es estadísticamente significativa $(F(1,165)=9,96, p<, 05)$. Este ejercicio permite apreciar que existe una brecha importante en la respuesta de las niñas, según si provienen de NSE alto o bajo (Gráfico 3). O sea, el hecho de pertenecer al NSE bajo hace que las niñas respondan con mayor intensidad con el estereotipo que asocia la matemática con el género masculino. En cambio, los niños varones asocian la matemática a lo masculino sin importar el NSE de origen. Adicionalmente, se puede interpretar que las niñas de nivel socioeconómico alto son las únicas de los cuatro grupos que no muestran una asociación entre el género y las matemáticas. 
Gráfico 3. ANOVA factorial género * NSE en medidas implícitas en niños/as

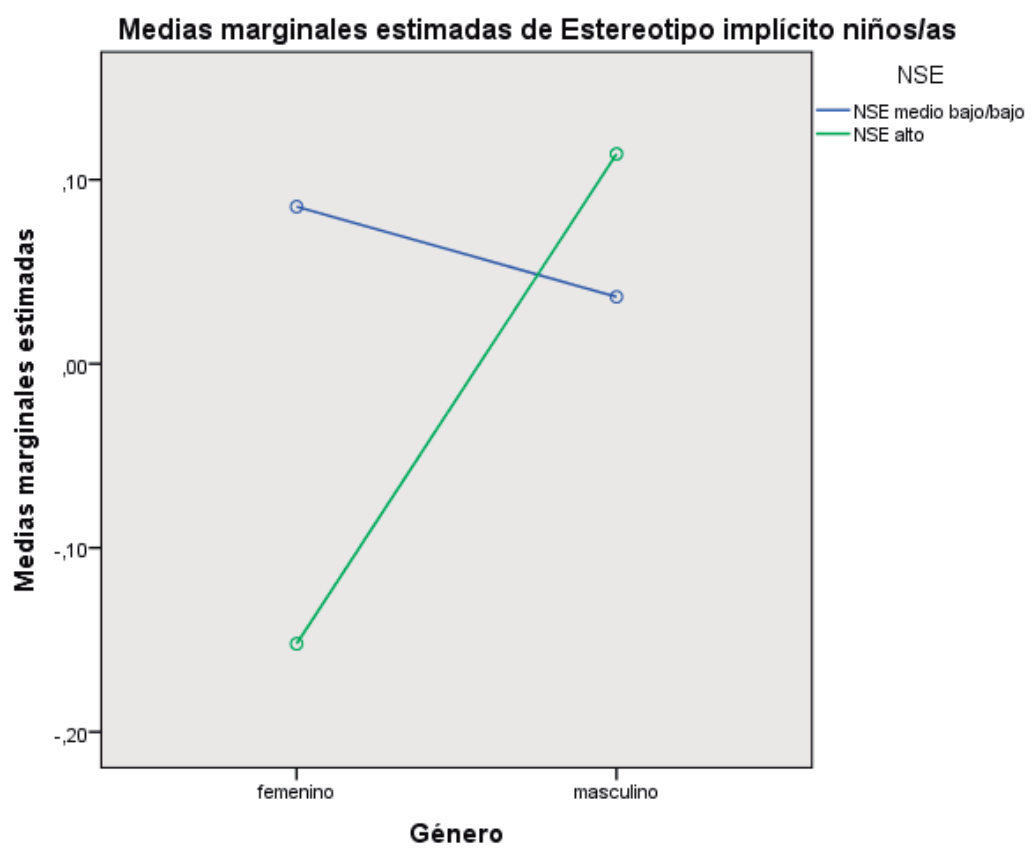

Además, al correlacionar los resultados de los niños y niñas en las pruebas de TEE e IAT, los resultados muestran que no lo hacen de manera significativa, lo que demuestra que las tendencias de respuesta son distintas, tal como se había ya mostrado en los análisis anteriores.

\section{Estereotipos de género en padres y madres}

Los resultados de los padres y madres en las pruebas de TEE e IAT se pueden apreciar en la Tabla 3. Al comparar datos de madres y padres, se puede apreciar que no existen diferencias estadísticas en las respuestas del TEE $(t(170)=1,57, p=, 117)$ o de IAT $(t(170)=, 08$, $p=$,936), o sea, padres y madres tienden a dar el mismo tipo de respuesta en ambos tests. Pero en todos los casos estas son estadísticamente distintas del valor 0 , o sea, tanto padres como madres en los test de TEE e IAT presentan respuestas que asocian la matemática al género masculino. Esto también ocurre al analizar sus respuestas en ambos tests de acuerdo con el NSE de pertenencia (Tabla 3). 
Tabla 3. Medias en medidas explícitas e implícitas en padres y madres y en subgrupos de NSE

\begin{tabular}{|c|c|c|c|c|c|c|c|c|c|c|c|c|}
\hline \multirow[t]{3}{*}{ Test } & \multicolumn{6}{|c|}{ Padres } & \multicolumn{6}{|c|}{ Madres } \\
\hline & \multicolumn{2}{|c|}{ Total } & \multicolumn{2}{|c|}{ NSE bajo } & \multicolumn{2}{|c|}{ NSE alto } & \multicolumn{2}{|c|}{ Total } & \multicolumn{2}{|c|}{ NSE bajo } & \multicolumn{2}{|c|}{ NSE alto } \\
\hline & $M$ & $D E$ & $M$ & $D E$ & $M$ & $D E$ & $M$ & $D E$ & $M$ & $D E$ & M & $D E$ \\
\hline TEE & $34^{*}$ &, 74 & $26^{*}$ &, 70 & $.43^{*}$ & .78 & $.46^{*}$ &, 70 &, $41^{*}$ &, 71 &, $52^{*}$ &, 70 \\
\hline IAT &, $21 *$ & .39 &, $20 *$ & .41 &, $21 *$ &, 38 &, $21^{*}$ &, 42 &, $25^{*}$ & .43 &, $15^{*}$ &, 40 \\
\hline
\end{tabular}

* Media estadísticamente distinta del valor $0 . p<, 01$.

Al analizar los resultados entre padres y madres de diferente NSE, es en el caso de las madres en el test IAT donde ocurre la única diferencia significativa. En este caso, las madres de NSE medio-bajo/ bajo -en comparación con las de NSE alto-asocian con una intensidad significativamente mayor la matemática con el género masculino $(t(169)=1,59, p<, 05$, unilateral), lo que se relaciona con los resultados antes revisados de los niños del mismo estrato socioeconómico.

Esto se puede observar en el Gráfico 4, donde se aprecia que las cajas correspondientes a las respuestas del test IAT de las madres de NSE medio-bajo/bajo se ubican, en su mayoría, por sobre el valor 0; mientras que las respuestas de las madres de NSE alto se encuentran más distribuidas en diferentes valores.

Gráfico 4. Resultado de medidas implícitas de estereotipos en madres según NSE

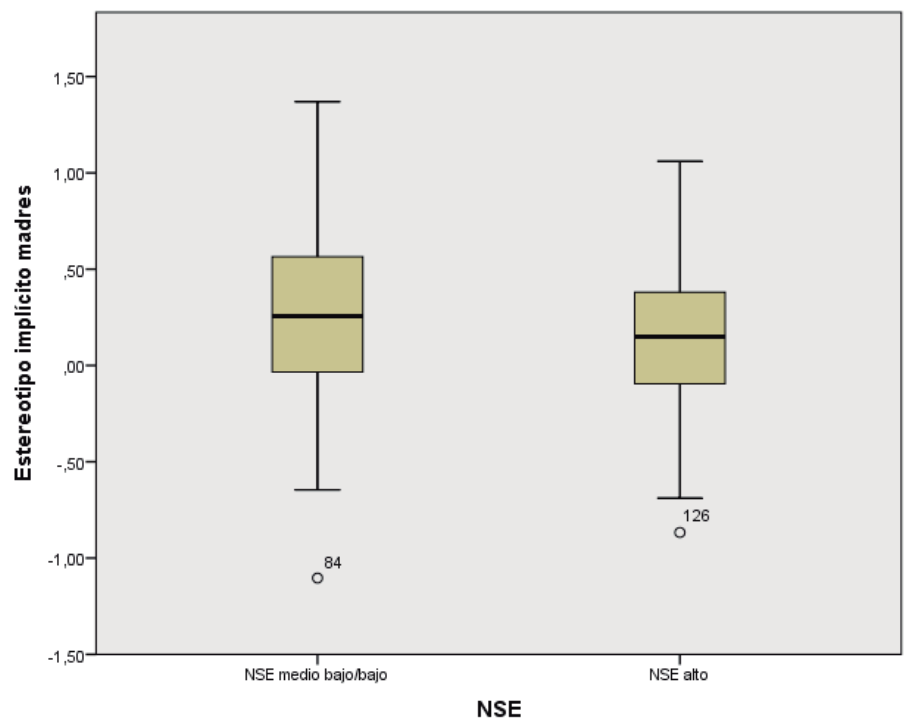


Al correlacionar los resultados de padres y madres en las pruebas de TEE e IAT los resultados muestran una correlación significativa entre los datos de pruebas explícitas e implícitas para ambos actores. De este modo el test TEE de las madres se asocia estadísticamente con sus resultados en el IAT $(r=, 173, p<, 05)$, y el mismo fenómeno ocurre con los datos de los padres $(r=, 198, p<, 01)$.

\section{Estereotipos de género en educadoras}

Las medias que las educadoras alcanzaron en los test de TEE e IAT se pueden apreciar en la Tabla 4.

Tabla 4. Medias en Medidas explícitas e implícitas de educadoras de acuerdo con NSE

\begin{tabular}{c|c|c}
\hline Test & \multicolumn{2}{|c}{ Total } \\
\hline & $M$ & $D E$ \\
\hline TEE &, $56^{*}$ &, 56 \\
\hline IAT &, $24^{*}$ &, 61 \\
\hline
\end{tabular}

* Media estadísticamente distinta del valor 0. $p<, 01$.

Al comparar las respuestas de las educadoras en general con el valor cero, todas las medias son significativamente mayores. Esto muestra que, como conjunto, las educadoras presentan un estereotipo que asocia la matemática al género masculino, tanto en medidas explícitas, como implícitas.

Dado que el número de educadoras $(n=19)$ es pequeño, no es recomendable llevar a cabo una comparación de resultados de acuerdo al NSE de las escuelas donde estas trabajan; pero sí es posible evaluar la relación de las respuestas con los dos tipos de medidas -TEE e IAT- de las educadoras. Este ejercicio muestra que presentan una alta correlación bivariada que resulta significativa $(r=, 353, p<, 01)$.

\section{Correlación entre las pruebas de los adultos y los niños}

Un último objetivo de este estudio buscó establecer el nivel de relación entre las creencias explícitas e implícitas de los niños y las de sus 
padres y educadoras. Al llevar a cabo este tipo de análisis se observa que no existen correlaciones significativas entre los resultados de los niños (en ambos test) y los resultados de madres, padres o educadoras.

Al realizar este mismo ejercicio separando los datos de acuerdo con el NSE de origen, solo se aprecia una correlación significativa, la que se da en los datos de TEE de los niños y niñas y los datos del mismo test de los padres en el NSE alto $(r=, 264, p<, 05)$. Al separar los datos de acuerdo con el género de los niños, se aprecia también una correlación significativa, esta vez una correlación negativa entre la medida TEE de las madres y el IAT de los niños hombres $(r=-, 293, p<, 01)$.

\section{Discusión}

Este estudio buscaba conocer los estereotipos de género relativos a habilidades matemáticas en niños y niñas, educadoras, madres y padres, evaluando posibles diferencias de acuerdo con el NSE de origen. Los datos muestran un estereotipo generalizado entre los adultos (padres, madres y educadoras) que asocia la matemática con el género masculino. Esta es una asociación que se mostró estadísticamente significativa en todos los casos y que demuestra que las hipótesis de trabajo estaban en la dirección correcta. Así, las brechas de resultados entre hombres y mujeres en las pruebas estandarizadas de matemática en el país tienen, como la otra cara de la moneda, el que los adultos más relevantes en la vida de los niños efectivamente creen que la matemática es un asunto que se asocia más con lo masculino que a lo femenino.

Estas creencias estereotipadas podrían producir tratamientos diferenciales de niños y niñas, los que a la vez transmiten mensajes distintos acerca de sus habilidades y generan diferentes oportunidades de aprendizajes. Por ejemplo, Nürnberger, Nerb, Schmitz, Keller y Sütterlin (2016), en un estudio realizado en Alemania, encontraron que los profesores recomendaban diferentes tipos de escuelas secundarias a los niños y a las niñas (los niños recibían recomendaciones de escuelas de orientación científico/matemática y las niñas, escuelas de orientación humanista). Aún más interesante, 
el grado en que las recomendaciones de un profesor se adherían al estereotipo de la matemática como algo masculino, era predicho por sus estereotipos implícitos de género y matemática, y por sus creencias de determinismo genético. Este es un ejemplo dramático de la manera en que los estereotipos de los adultos pueden crear ambientes de aprendizaje matemático muy distintos para niños y niñas. Otros comportamientos de los adultos pueden ser más sutiles, pero aun así crear brechas en las oportunidades de aprendizaje y el autoconcepto matemático. Por ejemplo y, en relación con los padres, Bhanot y Jovanovic (2005) realizaron un estudio acerca de los comportamientos de los padres de 38 estudiantes de $5^{\circ}$ a $8^{\circ}$ básico en EE.UU. al ayudarlos con sus tareas de matemáticas. En el estudio, el nivel de intrusividad — tendencia a proporcionar ayuda no solicitada - medió la relación entre los estereotipos parentales sobre género y matemática, y la percepción de las niñas de su propia habilidad, lo que sería consistente con la existencia de una cadena causal desde los estereotipos parentales al autoconcepto matemático, a través de las conductas de los padres. En otro estudio, Halpern y Perry-Jenkins (2016) midieron las conductas y creencias en relación con el género de 121 padres y madres en EE.UU. durante el primer año de vida de sus hijos e hijas, y encontraron que las conductas explicaban mejor las creencias estereotipadas de género de los niños y niñas de seis años. Este resultado es consistente con un modelo mediacional en que las creencias de los padres afectan a los niños a través de las conductas de los primeros.

En el presente estudio se ha visto que tanto educadoras como padres y madres poseen estos estereotipos y, por ende, se puede suponer que desde temprano en la vida de los niños y niñas que aún no entran a la educación básica podrían ofrecer diferentes oportunidades de aprendizaje. Como resultado, a medida que los estudiantes avancen en su educación, irán recibiendo oportunidades de aprendizaje de matemática diferenciadas por género, lo que aumentaría sus brechas de aprendizaje y rendimiento en esta disciplina.

Otro resultado que concuerda con lo esperado, es que todas las medidas de estereotipos correlacionan entre sí en el caso de los adultos. Esto quiere decir que el estereotipo está presente tanto 
a nivel implícito, como explícito, lo que otorga aún más fuerza a estas creencias. Además, estos hallazgos entregan evidencia de la consistencia de estas creencias estereotipadas de los adultos significativos en la vida de los niños.

A partir de lo anterior, se podría esperar que todos los niños presentaran el mismo fenómeno. Los resultados mostraron que todos los niños varones y las niñas de NSE bajo asociaban la matemática con lo masculino, cuando se evaluaba con instrumentos de corte implícito. En cambio, las niñas de NSE alto no asociaban la matemática a ningún género específico. Este fenómeno es de gran interés, pues muestra que estos estereotipos son creencias que se desarrollan alrededor de esta edad y que parecieran instalarse de manera diferenciada en distintos grupos. En efecto, estudios (Cvencek, Meltzoff et al., 2011) muestran que en niños estadounidenses estas creencias aparecen ya desde $2^{\circ}$ básico, tanto en hombres como mujeres. Nuestros resultados sugieren que en nuestro país aparecen desde más temprano y, que además, esta es una edad crítica donde intervenir.

Por otra parte, el hecho de que los estereotipos de los niños solo aparecieran con las medidas implícitas, no es de extrañar. Este tipo de instrumento ha demostrado ser más sensible a estos fenómenos, por lo cual es posible que, si los estereotipos están emergiendo de forma incipiente a los cinco años, solo los instrumentos más sensibles son capaces de detectarlos. En efecto, existe evidencia que muestra que los estereotipos de género infantiles se fortalecen con el paso del tiempo (Coyne, Linder, Rasmussen, Nelson \& Birkbeck, 2016, en prensa), por lo cual podemos entender los resultados obtenidos como una fotografía de un momento en el desarrollo donde los estereotipos de estos niños y niñas están lo suficientemente consolidados para afectar su conducta en pruebas implícitas, pero no lo bastante como para ser expresados en forma verbal. Asimismo, es importante destacar que este es un estudio pionero en evaluar con medidas implícitas y explícitas estereotipos en niños de tan corta edad, por lo que estos resultados contribuyen al cuerpo de conocimientos del desarrollo de los estereotipos en niños en edad preescolar. 
La diferencia en estereotipos que aparece entre los distintos niveles socioeconómicos también se había hipotetizado. Como ya se ha mencionado, en Chile la brecha de género en matemática es más marcada entre los estudiantes de menor ingreso. Los resultados son concordantes con esta realidad, pues justamente muestran que son las niñas y las madres de NSE bajo quienes presentan de manera más intensa el estereotipo. De esto se desprende que es justamente este grupo el que debiera ser prioritario a la hora de diseñar políticas que intenten ofrecer iguales oportunidades de aprendizaje a niños y niñas.

En resumen, la evidencia de este estudio que muestra que tanto adultos significativos -padres, madres y educadoras-, como niños de kínder ya poseen estereotipos que asocian la matemática a los hombres llama urgentemente a buscar intervenciones que se dirijan a promover que tanto niños como niñas tengan las mismas oportunidades para aprender y acceder a profundizar de las áreas que utilizan la matemática.

Algunos desafíos futuros son conocer la relación específica diferencial que los estereotipos de padres y madres tienen sobre los niños, de acuerdo con su género y llevar a cabo análisis que permitan explorar la relación de estereotipos de las educadoras con los niños anidados en su curso. Asimismo, sería relevante evaluar el efecto de los estereotipos de educadoras, madres y padres en el desarrollo de habilidades matemáticas tempranas y posteriores, de modo de estudiar su estabilidad y cambio en la transición a $1^{\circ}$ básico, donde los niños se ven expuestos a otros profesores y su autoconcepto se vuelve más estable.

\section{Referencias}

Alexander, G. M., Wilcox, T., \&Woods, R. (2009). Sex differences in infants visual interest in toys. Archives of Sex Behavior, 38(3), 427-433. http:// dx.doi.org/10.1007/s10508-008-9430-1

Ambady, N., Shih, M., Kim, A., \& Pittinsky, T. (2001). Stereotype susceptibility in children: Effects of identity activation on quantitative performance. Psychological Science, 5(12), 385-390. http://dx.doi.org/10.1111/14679280.00371 
Aronson, E., Wilson, T., \& Akert, R. (2010). Social psychology. New Jersey: Upper Saddle River.

Baron, A. S., Schmader, T., Cvencek, D., \& Meltzoff, A. N. (2014). The gendered self-concept: How implicit gender stereotypes and attitudes shape self-definition. En P. J. Leman \& H. R. Tenenbaum (Eds.), Gender and development (pp. 109-132). East Sussex, England: Psychology Press.

Berg, D. H. \& Klinger, D. A. (2009). Gender differences in the relationship between academic self-concept and self-reported depressed mood in school children. Sex Roles, 7-8(61), 501-509. http://dx.doi.org/10.1007/ s11199-009-9652-2

Bhanot, R. \& Jovanovic, J. (2005). Do parents' academic gender stereotypes influence whether they intrude on their children's homework? Sex Roles, 9-10(52), 597-607. http://dx.doi.org/10.1007/s11199-0053728-4

Bigler, R. S. \& Liben, L. S. (2007). Developmental intergroup theory explaining and reducing children's social stereotyping and prejudice. Current Directions in Psychological Science, 3(16), 162-166. http://dx.doi. org/10.1111/j.1467-8721.2007.00496.x

Blázquez, C., Álvarez, P., Bronfman, N., y Espinosa, J. (2009). Factores que influencian la motivación de escolares por las áreas tecnológicas e ingeniería. Calidad en la Educación, 31, 46-64.

Cárdenas, M. y Barrientos, J. (2008). Actitudes explícitas e implícitas hacia los hombres homosexuales en una muestra de estudiantes universitarios en Chile. Psykhe, 2(17), 17-25. http://dx.doi.org/10.4067/S071822282008000200002

Cerda, G., Pérez, C., \& Ortega-Ruiz, R. (2014). Relationship between early mathematical competence, gender and social background in Chilean elementary school population. Anales de Psicología, 3(30), 1006-1013. http://dx.doi.org/10.6018/analesps.30.3.152891

Cherney, I. D. \& London, K. (2006). Gender-linked differences in toys, television shows, computer games, and outdoor activities of 5 to 13 year old children. Sex Roles, 9-10(54), 717-726. http://dx.doi.org/10.1007/ s11199-006-9037-8

Consejo Nacional de Educación, CNED. (2005). Educación superior y género: tendencias observadas. Recuperado desde http://www.cned.cl/public/ secciones/SeccionPublicaciones/Publicaciones_Ver_art.aspx?idPub=5 2\&idArt=475\&nomArt=EducaciónSuperior y Género. Tendencias Ob servadas. \&nomPub=EstudiosyDocumentos. 
Coyne, S., Linder, R., Rasmussen, E., Nelson, D., \& Collier, K. (2014). It's a bird! It's a plane! It's a gender stereotype!: Longitudinal associations between superhero viewing and gender stereotyped play. Sex Roles, 9-10(70), 416-430. http://dx.doi.org/10.1007/s11199-014-0374-8

Coyne, S. M., Linder, J. R., Rasmussen, E. E., Nelson, D. A., \& Birkbeck, V. (2016, en prensa). Pretty as a princess: Longitudinal effects of engagement with Disney princesses on gender stereotypes, body esteem, and prosocial behavior in children. Child Development. http:// dx.doi.org/10.1111/cdev.12569

Cvencek, D., Greenwald, A., \& Meltzoff, A. (2011). Measuring implicit attitudes of 4-year-olds: The preschool implicit association test. Journal of Experimental Child Psychology, 2(109), 187-200. http://dx.doi. org/10.1016/j.jecp.2010.11.002

Cvencek, D., Kapur, M., \& Meltzoff, A. N. (2015). Math achievement, stereotypes, and math self-concepts among elementary-school students in Singapore. Learning and Instruction, 39, 1-10. http://dx.doi. org/10.1016/j.learninstruc.2015.04.002

Cvencek, D., Meltzoff, A., \& Greenwald, A. (2011). Math-Gender stereotypes in elementary school children. Child Development, 3(82), 766-779. http://dx.doi.org/10.1111/j.1467-8624.2010.01529.x

Cvencek, D., Meltzoff, A., \& Kapur, M. (2014). Cognitive consistency and math-gender stereotypes in Singaporean children. Journal of Experimental Child Psychology, 117, 73-91. http://dx.doi.org/10.1016/j. jecp.2013.07.018

Davies, P. G., Spencer, S. J., \& Steele, C. M. (2005). Clearing the air: Identity safety moderates the effects of stereotype threat on women's leadership aspirations. Journal of Personality and Social Psychology, 2(88), 276-287. http://dx.doi.org/10.1037/0022-3514.88.2.276

Del Río, M. F. \& Strasser, K. (2013). Preschool children's beliefs about gender differences in academic skills. Sex Roles, 3-4(34), 231-238. http:// dx.doi.org/10.1007/s11199-012-0195-6

Dunham, Y., Baron, A. S., \& Banaji, M. R. (2006). From American city to Japanese village: A cross-cultural investigation of implicit race attitudes. Child Development, 5(77), 1268-1281. http://dx.doi.org/10.1111/ j.1467-8624.2006.00933.x

Fazio, R. H. \& Olson, M. A. (2003). Implicit measures in social cognition research: Their meaning and use. Annual Review of Psychology, 1(54), 297-327. http://dx.doi.org/10.1146/annurev.psych.54.101601.145225 
Fennema, E., Peterson, P. L., Carpenter, T., \& Lubinski, C. (1990). Teacher's attributions and beliefs about girls, boys, and mathematics. Educational Studies in Mathematics, 1(21), 55-69. http://dx.doi.org/10.1007/ bf00311015

Fernández, J., Quiroga, M. A., Escorial, S., \& Privado, J. (2014). Explicit and implicit assessment of gender roles. Psicothema, 26, 244-251. http:// dx.doi.org/ 10.7334/psicothema2013.219

Fredricks, J. A. \& Eccles, J. S. (2002). Children's competence and value beliefs from childhood through adolescence: Growth trajectories in two malesex typed domains. Developmental Psychology, 4(38), 519-533. http:// dx.doi.org/10.1037//0012-1649.38.4.519

Galdi, S., Cadinu, M., \& Tomasetto, C. (2014). The roots of stereotype threat: When automatic associations disrupt girls' math performance. Child development, 1(85), 250-263. http://dx.doi.org/10.1111/cdev.12128

Greenwald, A. G. \& Banaji, M. R. (1995). Implicit social cognition: Attitudes, self-esteem, and stereotypes. Psychological Review, 1(102), 4-27. http:// dx.doi.org/10.1037/0033-295x.102.1.4

Greenwald, A. G., Banaji, M. R., Rudman, L. A., Farnham, S. D., Nosek, B. A., \& Mellott, D. S. (2002). A unified theory of implicit attitudes, stereotypes, self-esteem, and self-concept. Psychological Review, 1(109), 3-25. http://dx.doi.org/10.1037//0033-295x.109.1.3

Greenwald, A. G., McGee, D. E., \& Schwartz, J. L. (1998). Measuring individual differences in implicit cognition: The Implicit Association Test. Journal of Personality and Social Psychology, 6(74), 1469-1480. http://dx.doi.org/10.1037/0022-3514.74.6.1464

Greenwald, A. G., Nosek, B. A., \& Banaji, M. R. (2003). Understanding and using the implicit association test: I. An improved scoring algorithm. Journal of Personality and Social Psychology, 2(85), 197-216. http:// dx.doi.org/10.1037/0022-3514.85.2.197

Gunderson, E., Ramírez, G., Levine, S., \& Beilock, S. (2011). The role of parents and teachers in the development of gender-related math attitudes. Sex Roles, 3-4(66), 153-166. http://dx.doi.org/10.1007/ s11199-011-9996-2

Halpern, H. P. \& Perry-Jenkins, M. (2016). Parents' gender ideology and gendered behavior as predictors of children's gender-role attitudes: A longitudinal exploration. Sex Roles, 11-12(74), 527-542. http://dx.doi. org/10.1007/s11199-015-0539-0

Herbert, J. \& Stipek, D. (2005). The emergence of gender differences in children's perceptions of their academic competence. Journal of Applied 
Developmental Psychology: An International Lifespan Journal, 3(26), 276295. http://dx.doi.org/10.1016/j.appdev.2005.02.007

Hofmann, W., Gawronski, B., Gschwender, T., Le, H., \& Schmitt, M. (2005). A meta-analysis of the correlation between the implicit association test and explicit self-report measures. Personality and Social Psychology Bulletin, 10(31), 1369-1385. http://dx.doi.org/10.1177/0146167205275613

Jacobs, J. E. (1991). Influence of gender stereotypes on parent and child mathematics attitudes. Journal of Educational Psychology, 4(83), 518527. http://dx.doi.org/10.1037/0022-0663.83.4.518

Keller, C. (2001). Effect of teacher's stereotyping on students stereotyping of mathematics as a male domain. Journal of Social Psychology, 2(141), 165-173. http://dx.doi.org/10.1080/00224540109600544

Kiefer, A. \& Sekaquaptewa, D. (2007). Implicit stereotypes, gender identification, and math related outcomes: A prospective study of female college students. Psychological Science, 1(18), 13-18. http:// dx.doi.org/10.1111/j.1467-9280.2007.01841.x

Kurtz-Costes, B., Rowley, S. J., Harris-Britt, A., \& Woods, T. A. (2008). Gender stereotypes about mathematics and science and self-perceptions of ability in late childhood and early adolescence. Merrill Palmer Quarterly, 3(54), 386-409. http://dx.doi.org/10.1353/mpq.0.0001

Leahey, E. \& Guo, G. (2001). Gender differences in mathematical trajectories. Social Forces, 2(80), 713-732. http://dx.doi.org/10.1353/sof.2001.0102

LeFevre, J., Skwarchuk, S. L., Smith-Chant, B. L., Fast, L., Kamawar, D., \& Bisanz, J. (2009). Home numeracy experiences and children's math performance in the early school years. Canadian Journal of Behavioural Science, 2(41), 55-66. http://dx.doi.org/10.1037/a0014532

Levy, S. R. \& Killen, M. (Eds.). (2008). Intergroup attitudes and relations in childhood through adulthood. Oxford: Oxford University Press.

Lindberg, S., Linkersdörfer, J., Ehm, J., Hasselhorn, M., \& Lonnemann, J. (2013). Gender differences in children's math self-concept in the first years of elementary school. Journal of Education and Learning, 3(2), 1-7. http://dx.doi.org/10.5539/jel.v2n3pl

Lummis, M. \& Stevenson, H. (1990). Gender differences in beliefs and achievement: A cross-cultural study. Developmental Psychology, 2(26), 254-263. http://dx.doi.org/10.1037/0012-1649.26.2.254

Maccoby, E. E. (2002). Gender and group process: A developmental perspective. Current Directions in Psychological Science, 2(11), 54-58. http://dx.doi.org/10.1111/1467-8721.00167 
Madero, I. (2011). Inclusión y exclusión de género y clase al interior de la escuela chilena en 4 comunas del sur de Chile. Estudios Pedagógicos, 2(37), 135145. http://dx.doi.org/10.4067/S0718-07052011000200008

Marsh, H. W., Trautwein, U., Ludtke, O., Koller, O., \& Baumert, J. (2005). Academic self-concept, interest, grades, and standardized test scores: Reciprocal effects models of causal ordering. Child Development, 2(76), 397-416. http://dx.doi.org/10.1111/j.1467-8624.2005.00853.x

Martin, C. L. \& Ruble, D. (2004). Children's search for gender cues cognitive perspectives on gender development. Current Directions in Psychological Science, 2(13), 67-70. http://dx.doi.org/10.1111/j.09637214.2004.00276.x

Martin, C. L., Ruble, D. N., \& Szkrybalo, J. (2002). Cognitive theories of early gender development. Psychological Bulletin, 6(128), 903-933. http:// dx.doi.org/10.1037/0033-2909.128.6.903

Meece, J. L., Glienke, B. B., \& Burg, S. (2006). Gender and motivation. Journal of School Psychology, 44, 351-373.

Ministerio de Educación de Chile, Mineduc. (2011). Noticias. Recuperado de http://www.mineduc.cl/index2.php?id_portal=1\&id_seccion=10\&id_ contenido $=13142$.

Mizala, A., Martínez, F., \& Martínez, S. (2015). Pre-service elementary school teachers' expectations about student performance: How their beliefs are affected by their mathematics anxiety and student's gender. Teaching and Teacher Education, 50, 70-78. http://dx.doi.org/10.1016/j. tate.2015.04.006

Nosek, B., Smyth. F., Sriram, N., \& Lindner, N. (2009). National differences in gender-science stereotypes predict national sex differences in science and math achievement. Proceedings of the National Academy of Sciences of the United States of America, 26(106), 10593-10597. http://dx.doi. org/10.1073/pnas.0809921106

Nürnberger, M., Nerb, J., Schmitz, F., Keller, J., \& Sütterlin, S. (2016). Implicit gender stereotypes and essentialist beliefs predict preservice teachers' tracking recommendations. Journal Of Experimental Education, 1(84), 152-174. http://dx.doi.org/10.1080/00220973.2015.1027807

Organisation for Economic Co-operation and Development, OECD. (2010). PISA 2009 Results: Executive Summary. Recuperado de http://www. oecd.org/dataoecd/34/60/46619703.pdf.

Organisation for Economic Co-operation and Development, OECD (2014). PISA 2012 Results: What students know and can do. Recuperado de http:// www.oecd.org/pisa/keyfindings/pisa-2012-results-volume-I.pdf. 
Organisation for Economic Co-operation and Development, OECD (2015). "Chile" in Education at a Glance 2015. OECD Indicators. Paris: Autor.

Rutland, A., Cameron, L., Milne, A., \& McGeorge, P. (2005). Social norms and self-presentation: Children's implicit and explicit intergroup attitudes. Child Development, 2(76), 451-466. http://dx.doi.org/10.1111/j.14678624.2005.00856.x

Schwartz, C. S. \& Sinicrope, R. (2013). Prospective elementary teachers' perceptions of gender differences in children's attitudes toward mathematics. School Science and Mathematics, 8(113), 410-416. http:// dx.doi.org/10.1111/ssm. 12045

Servicio Nacional de la Mujer, Sernam. (2008). Análisis de género en el aula. Recuperado de http://estudios.sernam.cl/documentos/?eMTEONDczN w==Análisis_de_Genero_en_el_Aula.

Siebler, F., González, R., Ordóñez, G., Bohner, G., Haye, A., Sirlopú, D., ... \& Torres, D. (2010). The category-focus implicit association test. Social Psychology, 2(41), 105-110. http://dx.doi.org/10.1027/18649335/a000015

Sirlopú, D., González, R., Bohner, G., Siebler, F., Millar, A., Ordóñez, G., ... $\&$ de Tezanos-Pinto, P. (2012). Actitudes implícitas y explícitas hacia personas con Síndrome de Down: un estudio en colegios con y sin programas de integración de Chile. Revista de Psicología Social, 2(27), 199-210. http://dx.doi.org/10.1174/021347412800337861

Sistema de Medición de la Calidad de la Educación, Simce. (2012). Resultados nacionales Simce 2011. Recuperado de http://www.agenciaeducacion. cl/wpcontent/files_mf/infornenacional2011_web.pdf

Sistema de Medición de la Calidad de la Educación, Simce. (2013). Informe Nacional de Resultados Simce 2012. Recuperado de https:// s3-us-west-2.amazonaws.com/documentos-web/Informes/ Informe+Nacional+Simce+2012.pdf

Skaalvik, S. \& Skaalvik, E. M. (2004). Gender differences in math and verbal selfconcept, performance expectations, and motivation. Sex Roles, 3-4(50), 241-252. http://dx.doi.org/10.1023/b:sers.0000015555.40976.e6

Sonnenschein, S., Galindo, C., Metzger, S., Thompson, J., Huang, H., \& Lewis, H. (2012). Parent's beliefs about children's math development and children's participation in math activities. Child Development Research, 2012, 1-13. http://dx.doi.org/10.1155/2012/851657

Steele, C. M. \& Aronson, J. (1995). Stereotype threat and the intellectual test performance of African Americans. Journal of personality and social Psychology, 5(69), 797-811. http://dx.doi.org/10.1037//00223514.69.5.797 
Steele, C. M., Spencer, S. J., \& Aronson, J. (2002). Contending with group image: The psychology of stereotype and social identity threat. Advances in Experimental Social Psychology, 34, 379-440. http://dx.doi. org/10.1016/s0065-2601(02)80009-0

Teachman, B. A., Gregg, A. P., \& Woody, S. R. (2001). Implicit associations for fear-relevant stimuli among individuals with snake and spider fears. Journal of Abnormal Psychology, 2(110), 226-235. http://dx.doi. org/10.1037/0021-843X.110.2.226

Tiedemann, J. (2000a). Gender-related beliefs of teachers in elementary school mathematics. Educational Studies in Mathematics, 2(41), 191-207. http:// dx.doi.org/10.1023/A:1003953801526

Tiedemann, J. (2000b). Parent's gender stereotypes and teacher's beliefs as predictors of children's concept of their mathematical ability in elementary school. Journal of Educational Psychology, 1(92), 144-151. http://dx.doi.org/10.1037/0022-0663.92.1.144

Tiedemnann, J. (2002). Teacher's gender stereotypes as determinants of teacher perceptions in elementary school mathematics. Educational Studies in Mathematics, 1(50), 49-62. http://dx.doi. org/10.1023/A:1020518104346

Tudge, J. R. \& Doucet, F. (2004). Early mathematical experiences: Observing young black and white children's everyday activities. Early Childhood Research Quarterly, 1(19), 21-39. http://dx.doi.org/10.1016/j. ecresq.2004.01.007

Walton G. M. \& Spencer, S. J. (2009). Latent ability: Grades and test scores systematically underestimate the intellectual ability of negatively stereotyped students. Psychological Science, 9(20), 1132-1139. http:// dx.doi.org/10.1111/j.1467-9280.2009.02417.x

Ware, N. \& Lee, V. (1986). When and why girls "leak" out of high school mathematics a closer look. Washington, D.C.: ERIC Clearinghouse.

Recibido: 26/07/2016

Aceptado: 01/10/2016 